Notfall Rettungsmed 2011 · 14:439-440

DOI 10.1007/s10049-011-1529-6

(c) Springer-Verlag 2011

\author{
U. Kreimeier ${ }^{1} \cdot$ H.-R. Arntz ${ }^{2}$ \\ ${ }^{1}$ Klinik für Anaesthesiologie, Klinikum der Universität München \\ ${ }^{2}$ Medizinische Klinik II, Kardiologie und Pulmonologie, \\ Campus Benjamin Franklin, Charité, Berlin
}

\title{
Der Tag, der die Welt veränderte
}

Bei dem Terroranschlag auf das World Trade Center in New York am 11. September 2001 kamen über 2900 Menschen ums Leben. Die Zahl der akut Verletzten wird auf über 6000 geschätzt.

Unabhängig von der gelegentlich wechselnden Einschätzung der aktuellen Gefährdungslage durch den Terror müssen wir uns vergegenwärtigen, dass in vielen deutschen (Groß-)Städten, und zwar vor allem an Wochenenden, Zehntausende an Großveranstaltungen teilnehmen. Jedes Mal sind von den Veranstaltern rettungsdienstliche Vorkehrungen zu treffen (• Infobox 1).

In die konkrete Gefahrenanalyse fließen u. a. die Besucherzahl, die Tatsache, ob die Veranstaltung in einem umschlossenen Raum oder im Freien stattfindet, und natürlich auch polizeiliche Erkenntnisse ein. Entsprechend der „Maurer-Formel“" werden z. B. bei einer Outdoorveranstaltung mit 40-50.000 zulässigen $\mathrm{Be}$ suchern 10 Punkte vergeben, bei 50.000 verkauften Tickets kommen 100 Punkte hinzu. Zusammen mit der „Gefahrenneigung " $z$. B. bei einem Rockkonzert (Multiplikator 1,0) ergeben sich 110 Punkte. Liegen polizeiliche Erkenntnisse mit Hinweisen auf Gewaltbereitschaft vor, kommen 1o Punkte hinzu (ebenso bei der fast schon obligaten Anwesenheit Prominenter: je 5 Prominente 10 Punkte). Das Gesamtrisiko liegt damit schnell bei weit über 100 Punkten. Unter anderem sind damit mehrere Notärzte (in dem Beispiel 5 Notärzte!) speziell für diese Veranstaltung vorzusehen. Es stellt sich dann vor allem die Frage: Wohin mit akut Erkrank- ten oder Verletzten? Hand aufs Herz: Wissen Sie in Ihrem Rettungsdienstbereich, welche Krankenhäuser im Ernstfall welche Zahl von Patienten mit welchem Verletzungsmuster aufzunehmen in der Lage sind?

Zur Weltmeisterschaft 2006 in Deutschland wurden in den Austragungsorten und umliegenden Rettungsdienstbereichen Alarmierungs- und Einsatzpläne speziell für den Massenanfall von Verletzten (MANV) erarbeitet. Das Krankenhauspersonal wurde geschult - wie sieht es 5 Jahre danach aus? Diese Frage gewinnt akut an Bedeutung, wenn große Veranstaltungen angekündigt sind.

Das diesjährige Oktoberfest 2011 in München begann am Samstag, den 17. September: Die Fernsehzuschauer können sehen, wie Oberbürgermeister Ude (fast) pünktlich um 12 Uhr das erste Fass ansticht und damit die Wiesn eröffnet. Bereits an diesem ersten Tag werden 400.00o Besucher erwartet, es ist Sonnenschein, bis zum frühen Abend herrschen $25^{\circ} \mathrm{C}$. Für traditionelle Oktoberfestbesucher ist eines gewiss: Bis 17 Uhr spätestens muss ich im Zelt sein, dann ist der Eingang zumeist geschlossen, da die Zelte voll sind. Dann ziehen Gewitterwolken auf und die meisten Besucher versuchen, aus dem Freien in die Zelte zu gelangen.

Noch eine halbe Woche zuvor war bei einer Einweisung für den Führungsdienst referiert worden, dass der Andrang auf der Wiesn punktuell so groß sein kann, dass hier, wenn es schlecht läuft, 4 Personen je Quadratmeter (!) stehen, sich über eine Viertelstunde und länger weder vor- wärts noch rückwärts bewegen können. Fast immer herrscht $20 \mathrm{~m}$ weiter keinerlei Gedränge - punktuell eben. Dies ist auf Fotoaufnahmen aus dem letzten Jahr von oben zu erkennen. Es genügt, wenn ein „Dummkopf“ bei einer derartigen Massenansammlung in einem Zelt Pfefferspray versprüht. Im Bericht des Rettungsdienstes vom Eröffnungstag liest sich das dann später so: „Reizgas, 37 Betroffene im Zelt“. Kein Wunder also, dass insgesamt an diesem Eröffnungssamstag weit über 300 Rettungsdiensteinsätze allein auf dem Gelände des Oktoberfests registriert werden. Es war offenbar auch klug geplant, dass neben den ständig im Stadtgebiet besetzten 11 Notarzteinsatzfahrzeugen und Notarztwagen an diesem Tag 4 weitere NEF für die Wiesn disponiert worden waren.

Beim Lagevortrag zur Unterrichtung des Einsatzführungsdienstes am nächsten Tag (18. September 2011) in der Integrierten Leitstelle referiert der Leiter des Stabes für Gefahrenabwehr über den erwarteten Besucherandrang (wiederum 400.000, davon in jedem Festzelt bis zu 10.00o Personen), die Wetterprognose (Starkregen) und weitere Veranstaltungen am selben Tag: Das Fußballspiel zwischen dem TSV 1860 München und dem FSV Frankfurt in der Allianz-Arena (zu den letzten Heimspielen kamen jeweils 25.00o Besucher, jetzt haben die 186oer in den letzten 3 Spielen 9 Punkte geholt...) sowie zwei weitere Veranstaltungen in Oberschleissheim und am Hinterbrühler See. Da erscheint die ebenfalls für diesen Tag angekündigte Veranstaltung eines prominenten Politikers beinahe als „Nebenbefund“. 


\section{Infobox 1}

Unglück mit einer Vielzahl von Verletzten durch plötzlich aufziehendes Unwetter, u. a. beim Pukkelpop-Festival in der Nähe von Hasselt in Belgien 2011. Hier finden Sie den QR-Code zum Filmbericht.

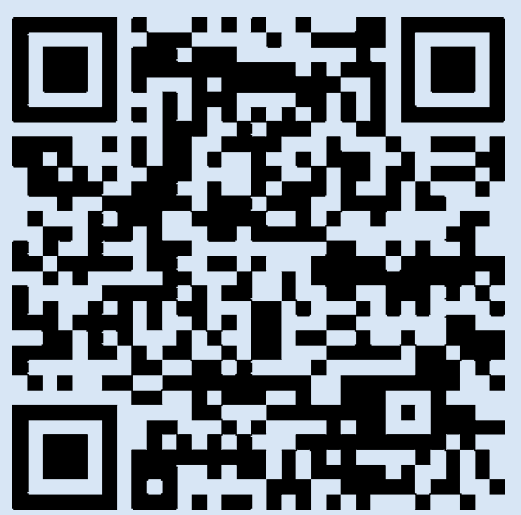

Countrykonzert in Indianapolis: Beim Einsturz einer Konzertbühne in den USA kamen 5 Menschen ums Leben, mindestens 45 wurden - z. T. schwer - verletzt. Zu dem Unglück kam es infolge eines plötzlichen, äußerst heftigen Windstoßes, der das Bühnengerüst zum Wanken brachte. Auf YouTube schildert ein Augenzeuge:

"I was there, in the Grandstands, and those winds were no where near a Tornado. Someone came out and told us that there is a storm coming, but they were going to continue the concert in a minute (as usual) and if it starts to rain we will go to the shelter areas and wait it out. The winds came out of no where. There was no time to stop the show and get everyone out."

Hier finden Sie den QR-Code zum Filmbericht.

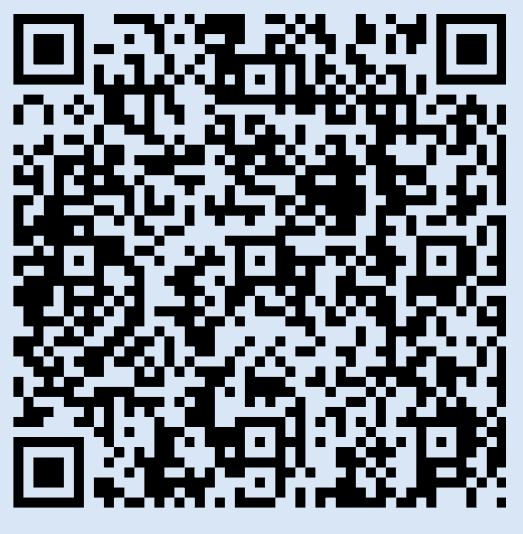

Spätestens hier stellt sich die Frage, ob im Fall eines größeren Unglücks bei einer Großveranstaltung, bei dem das Ausmaß in der Frühphase meist unbekannt ist, mit der typischen nachfolgenden Chaoswelle überhaupt rettungsdienstlich und katastrophentechnisch planbar, geschweige denn praktisch beherrschbar sein kann. Ganz entscheidend kommt es auf die Analyse im Vorfeld an (Gefahrenanalyse), die zu treffenden Vorkehrungen im Sinne von Fahrzeug- und Personalvorhaltung im Rettungsdienst, bei der Feuerwehr und allen anderen in der Gefahrenabwehr und im Katastrophenschutz beteiligten Stellen und Organisationen. Elementar scheint zudem die Kommunikation zu sein: Wie bekommt die Leitstelle schnellstmöglich ein „Bild“ von der Unglücksstelle, wie schnell muss der Rettungsdienst „hochgefahren" werden?

Das Mobilfunknetz kann man in diesen Fällen immer vergessen: Spätestens seit dem Bon-Jovi-Konzert im Münchner Olympiastadion in diesem Sommer (67.00o Besucher) ist bekannt, dass Veranstalter und Konzertbesucher bzw. über Mobilfunk zugeschaltete Teilnehmer die Mobilfunknetze komplett besetzen. Bei diesem Konzert konnte man wegen der hohen, nicht zu deckenden Nachfrage nach Tickets eine Zugangsberechtigung für eine Online-Übertragung des Konzerts im Internet kaufen. Was macht der pfiffige Konzertbesucher: Er überträgt das Konzert von seinem Mobilfunkgerät - sämtliche Mobilfunkzellen im Umfeld waren völlig ausgebucht, selbst mit Vorrangsberechtigung konnte man sich nicht einwählen. Diese Tatsache wurde erst jüngst wieder als Argument zur Unterstreichung der dringlichen Notwendigkeit zur Einführung des Digitalfunks in Bayern angeführt.

München ist nicht Deutschland. Es gibt größere Städte, zum Beispiel Berlin. Auch hier haben wir eine Mannschaft, die an jedem zweiten Wochenende in der Bundesliga spielt. Das Berliner Olympiastadion fasst 74.00o Besucher. Interessant erscheint in diesem Zusammenhang auch die Zahl von nur etwas über 800 PKWParkplätzen auf dem Gelände des Olympiastadions, womit der Hauptbesucheranund -abstrom über öffentliche Verkehrsmittel erfolgt. In der Hauptverkehrszeit eingesetzte S-Bahnen und U-Bahn-Züge befördern jeweils bis zu 8 oo Personen!

Auch in Berlin haben wir regelmäßig Großveranstaltungen mit z. T. mehreren zehntausend erwarteten Teilnehmern, die besondere Vorhaltungen auf Seiten des Rettungsdienstes erfordern. Oder Frank- furt, oder Hamburg,... Bedenken Sie bitte, dass allein 18 Vereine in der Bundesliga und 18 Vereine in der 2. Bundesliga spielen! Hierbei ist es unabdingbar, dass neben dem rettungsdienstlichen Einsatzgeschehen auch die Schnittstelle zu den Krankenhäusern analysiert wird und entsprechende Aufnahmekapazitäten vorgehalten bzw. kurzfristig bereitgestellt werden. Die Krankenhäuser müssen in die Konzepte des Rettungsdienst noch mehr eingebunden werden, da andernfalls das Chaos am Notfallort mit großer Sicherheit in die Krankenhäuser übertragen wird.

Sehr geehrte Leserinnen und Leser,

so schön sportliche und andere Großveranstaltungen auch für den Zuschauer sein können, für den Rettungsdienst stellen sie jedes Mal eine erneute Herausforderung dar. Die Besucherzahl ist gerade bei Open-Air-Veranstaltungen nur grob kalkulierbar, Programmabläufe können nicht $100 \%$ ig garantiert werden, Wetterprognosen sind nicht immer zuverlässig, und gegen die Macht der Elemente sind wir generell nicht gefeit! Ihre

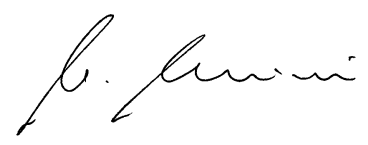

U. Kreimeier

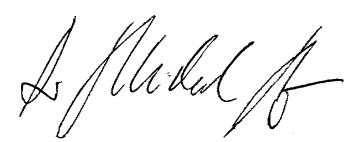

H.-R. Arntz

\section{Korrespondenzadresse}

\section{Prof. Dr. U. Kreimeier}

Klinik für Anaesthesiologie, Klinikum der Universität München

Nussbaumstraße 30, 80336 München Uwe.Kreimeier@med.uni-muenchen.de 